\title{
Revision Stapedotomies: The Role of Periprosthetic Scar Tissue Formation in the Development of Unsatisfactory Hearing Results after Stapedotomy
}

\author{
Gábor Polony ${ }^{10}$ Anita Gáborján ${ }^{10}$ László Tamás ${ }^{1}$ László Székely ${ }^{10}$ \\ ${ }^{1}$ Department of Otorhinolaryngology, Head and Neck Surgery, \\ Semmelweis Egyetem (Semmelweis University), Budapest, Hungary. \\ Int Arch Otorhinolaryngol 2022;26(3):e422-e427. \\ Address for correspondence László Székely, MD, Department of \\ Otorhinolaryngology, Head and Neck Surgery, Semmelweis Egyetem, \\ Szigony Str. 36, Budapest, 1083, Hungary \\ (e-mail: szekelyl@med.semmelweis-univ.hu).
}

\begin{abstract}
Introduction Revision stapes surgeries are difficult to perform, and their audiological results are inferior to primary surgeries.

Objective Our goal was to identify the most common and most influential postoperative reasons that cause persistent air-bone gap (ABG) after the primary surgery. Our focus was concentrated on the mechanical dysfunctions in the middle ear, with special regard to postoperative adhesion formation.

Methods We performed a retrospective case series study with 23 cases that underwent revision stapedotomies.

Results A significant improvement was seen in ABG and air conduction levels after surgery. The periprosthetic adhesion formation was seen in $65 \%$ of the cases, and it was the primary cause behind the unsatisfactory hearing result in $30 \%$ of cases. There was no significant difference in the level of persistent $A B G$ s after the primary surgery, in case of the intratympanic adhesion presence, compared with the presence of other surgical failures. Concerning hearing and $A B G$ gain after revision surgery, the noninferiority of the negative effect associated with adhesion was shown compared with the other reasons.

\section{Keywords}

- otosclerosis

- stapes

- stapedotomy

- otologic surgical techniques

- middle ear

Conclusion The revision stapedotomy is an efficient treatment option in case of persistent $A B G$. Periprosthetic adhesions are the most common intratympanic reasons for compromised audiological outcomes after stapedotomy.

Adhesion formations have the same negative effect on $A B G$ development as any other surgical failure, and the revision could be more challenging in these cases. These findings highlight the use of the most atraumatic surgical technique and preservation of intact intratympanic mucosa during middle ear surgery.
\end{abstract}

received

November 30, 2020 accepted after revision

September 2, 2021

published online

December 13, 2021
DOI https://doi.org/ $10.1055 / \mathrm{s}-0041-1740100$ ISSN 1809-9777.

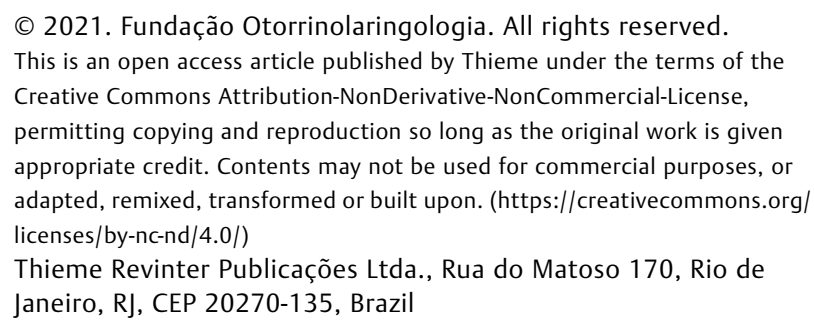

(c) 2021. Fundação Otorrinolaringologia. All rights reserved. This is an open access article published by Thieme under the terms of the Creative Commons Attribution-NonDerivative-NonCommercial-License, permitting copying and reproduction so long as the original work is given appropriate credit. Contents may not be used for commercial purposes, or adapted, remixed, transformed or built upon. (https://creativecommons.org/ licenses/by-nc-nd/4.0/) Thieme Revinter Publicações Ltda., Rua do Matoso 170, Rio de Janeiro, RJ, CEP 20270-135, Brazil 


\section{Introduction}

The stapedotomy is considered an efficient and safe surgical option in the treatment of stapes fixation. ${ }^{1}$ It has been shown that the postoperative air-bone gap (ABG) of $10 \mathrm{~dB}$ or less-which is defined as surgical success according to the American Academy of Otolaryngology, Head and Neck Surgery (AAO-HNS) guidelines ${ }^{2}$-could be achieved in 87 to $97 \%$ of the cases. ${ }^{3}$ Although this number is considered as a high success rate, it means that at least one revision is needed in 3 to $13 \%$ of the surgeries. According to available data, revision surgeries are generally more difficult to perform, and their audiological results are inferior to primary surgeries. ${ }^{4}$ The prevention of primary failure seems to be the most favorable option to achieve superior results. Revision surgeries offer the opportunity to recognize the exact reasons behind unsatisfactory results. According to previous data, intratympanic adhesion formation is one of the most common findings during revisions, ${ }^{5,6}$ but its influence on the hearing results is not clear yet. Our goal was to identify the most common and most influential postoperative causes for persistent $A B G$, and to clarify the relationship between the presence of adhesion and its impact on hearing. We focused on the mechanical dysfunctions in the middle ear, with special regard to postoperative adhesion formation. Our hypothesis was that postsurgical inflammation and scar tissue formation in the middle ear could be the main reasons behind compromised ABG gain.

\section{Materials and Methods}

\section{Patients}

A retrospective case series study of 23 revision stapedotomies was performed. The surgeries were performed between 2013 and 2020, at the Department of Otorhinolaryngology, Head and Neck Surgery of the Semmelweis University, Budapest, Hungary. During this period, 175 primary stapedotomies and 33 revision stapes surgery cases were noted, of which 10 cases were excluded due to incomplete follow-up data. Regarding the revision surgeries, the primary operation was performed by another surgeon in 13 cases. The indication for revision was $10 \mathrm{~dB}$ or higher persistent air-bone gap pure tone average (ABG-PTA). The average time between the primary and revision surgeries was 749 days (range: 8411,764 days). The presumed preoperative clinical diagnosis of stapes fixation before primary intervention was made by pure tone average (PTA) testing, tympanometry, stapedius reflex, and physical examination, and was confirmed with palpation during the surgery. Out of the 23 cases, 6 patients were male (26\%) and 17 were female (74\%), with an average age of 50.4 (standard deviation [SD] 12.0) years.

\section{Surgical Technique}

The same standardized surgical approach was applied and performed by the same senior surgeon in all of the cases. After an endaural approach, the posterior tympanomeatal (TM) flap was elevated, and the cause of failure was verified by the palpation of the ossicular chain and by microscopic examina- tion. According to the intratympanic findings, prosthesis replacement or repositioning was performed, and adhesions were removed mechanically. In five cases, a malleovestibulopexy was performed. The stapedectomy was performed in one case, otherwise, small fenestra technique was preferred. Most of the surgeries were performed under general anesthesia (19 cases, $82.6 \%)$, while in 4 cases (17.4\%) local anesthesia was preferred according to the patient's choice.

The prosthesis loop was bridged with a small connective tissue to prevent the slipping and necrosis of the long process. The adequate positioning and mobility of the ossicular chain and prosthesis were verified under microscope in each case at the end of the procedure. The TM flap was restored, the meatal incisions were overlaid with sterile silicone sheets, and the ear canal was stuffed with ofloxacin-impregnated, absorbable, hemostatic-gelatin sponge (Spongostan, Ethicon Somerville, $\mathrm{NJ}$, USA) pieces. The incision was closed with sutures.

The length of the applied prosthesis was $4.5 \mathrm{~mm}$ in $52 \%$ of the cases, $5 \mathrm{~mm}$ in $9 \%$, and $6 \mathrm{~mm}$ in $22 \%$ of the cases, with a diameter of $0.4 \mathrm{~mm}$ in $34 \%$ and $0.6 \mathrm{~mm}$ in $49 \%$ of the cases. The majority (48\%) of the applied pistons were stainless/ fluoroplastic Schuknecht Wire Pistons Slim Shaf (Gyrus Acmi Inc., Westborough, MA, USA). Richards Platinum Fluoroplastic Pistons (Olympus, Tokyo, Kanto, Japan) were applied in $35 \%$ of the cases. In the remaining surgeries $(17 \%)$, the original pistons were used.

\section{Methods}

In all cases, the exact reason behind the unsatisfactory primary results and the surgical solution were noted. The intratympanic problems were classified based on whether the failure was related to scar tissue formation, such as periprosthetic adhesion, inter- or periossicular adhesion (ossicular chain fixation), or any other specific surgeryrelated complication (long process of the incus [LPI] necrosis, prosthesis dislocation, ossicular luxation etc.). The scar tissue formation was considered as the main reason if it was the only finding during surgery.

In our definition, adhesion is an intratympanic scar tissue formation, considered as a fibrotic, non-anatomical connection between two tissue planes induced by injury.

The cases were divided into three groups: group I - no scar tissue present; group II - scar tissue present; and group III only scar tissue present. During the statistical evaluation, groups II and III were tested against group I.

The average postoperative follow-up time was 526 days (SD 521; range 92-1827 days). The cut-off point was at least 3 months of follow-up. These results could be interpreted as midterm results, but, according to the literature, they could be considered as an indicator of the long-term results. ${ }^{7}$

The air conduction (AC) levels were measured at 0.125 ; $0.25 ; 0.5 ; 1 ; 2 ; 4$; and $8 \mathrm{kHz}$, and the bone conduction (BC) was registered at $0.25 ; 0.5 ; 1 ; 2$; and $4 \mathrm{kHz}$. The evaluation was done according to the Committee on Hearing and Equilibrium guidelines of the AAO-HNS. ${ }^{2}$ The thresholds were reported, and the extended PTA was calculated from $0.5 ; 1 ; 2 ; 3 \mathrm{kHz}$ frequencies. The $3 \mathrm{kHz}$ values were 
considered as the mean of 2 and $4 \mathrm{kHz}{ }^{8}$ The ABG was calculated from same-time measurements. These data were retrospectively analyzed statistically (IBM SPSS Statistics for Windows, Version 25.0, Armonk, NY, US) to define descriptive data and the difference between types of surgical failure. The bootstrapped independent samples $t$-test was used for analyzing group differences, while intervention success was examined with the paired samples $t$-test. The two one-sided $t$-tests (TOST) with the effect size of ' 1 ' were used to determine equivalence and non-inferiority (TIBCO Statistica, Palo Alto, CA, US). The statistical significance level was $p<0.05$.

\section{Results}

The average postoperative AC-PTA was $46.9 \mathrm{~dB}$, which is significantly better compared with the $60.7 \mathrm{~dB}$ preoperative value $(p=0.003)$. After surgical intervention, ABG-PTA also improved significantly: $17.2 \mathrm{~dB}$ vs $31.1 \mathrm{~dB}(p<0.001)$. No significant difference could be seen between pre and postoperative BC-PTAs: $29.6 \mathrm{~dB}$ vs $29.6 \mathrm{~dB}(p=0.984)$ (-Fig. 1.a). The minimum of $30 \mathrm{~dB}$ AC-PTA was achieved in $39 \%$ of the cases. In $52.2 \%$ of the cases, at least $10 \mathrm{~dB} A B G$ gain was achieved, while the $10 \mathrm{~dB}$ or better $\mathrm{ABG}$ closure was achieved in $43.5 \%$. Finally, ABG closure between 10 and $20 \mathrm{~dB}$ prevailed in $13 \%$ of the cases (-Fig. 1.b).

Intratympanic adhesion was the most common finding during revision surgeries, with a prevalence of $65 \%$, and, in $30 \%$ of the cases, it was the main reason behind compromised ossicular chain mobility. Among the $30 \%$ ( 8 patients) of cases in which the only abnormal intratympanic finding was scar tissue, there were 6 cases with satisfactory hearing gain after the first intervention, which was followed by a progressive conductive hearing loss. In the remaining $35 \%$ of patients, the main problem was due to other surgical failures, while in $35 \%$ we found a combination of two factors (- Fig. 2.). The most common prosthesis-related complication was the disconnection between the piston loop and the LPI. According
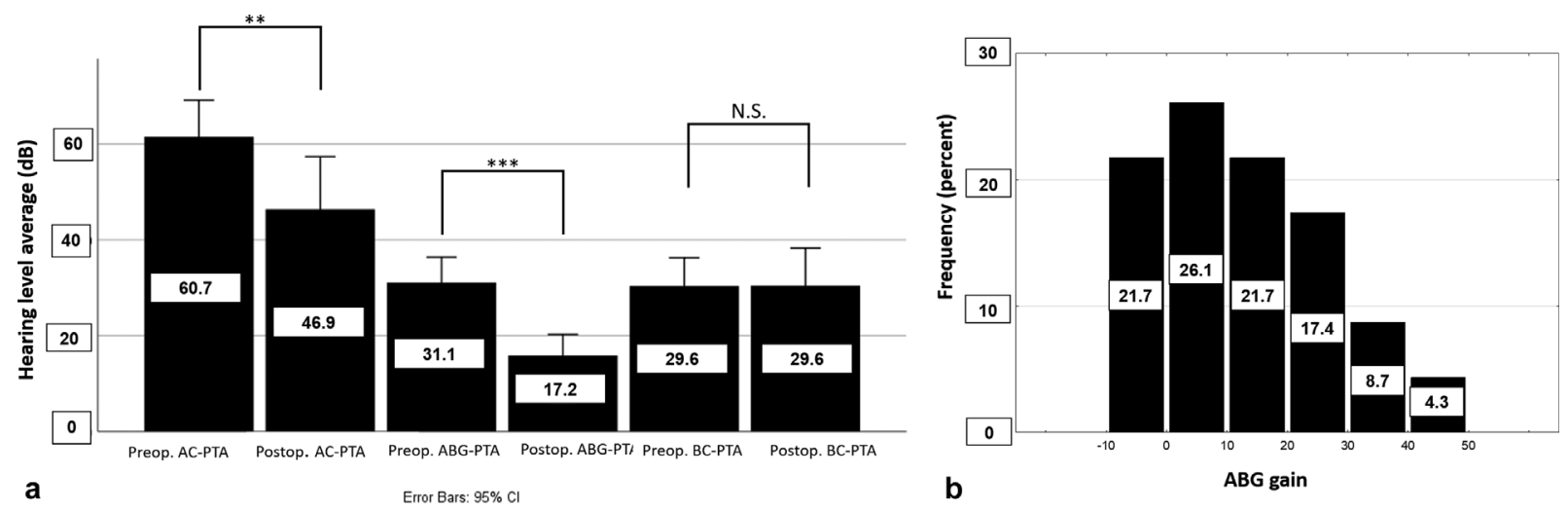

Fig. 1 (a) Pre and postoperative pure tone audiometry air-conduction hearing thresholds. Significant improvement after revision surgery. (b) Distribution of $A B G$ gain after revision surgery in $10 \mathrm{~dB}$ bins.

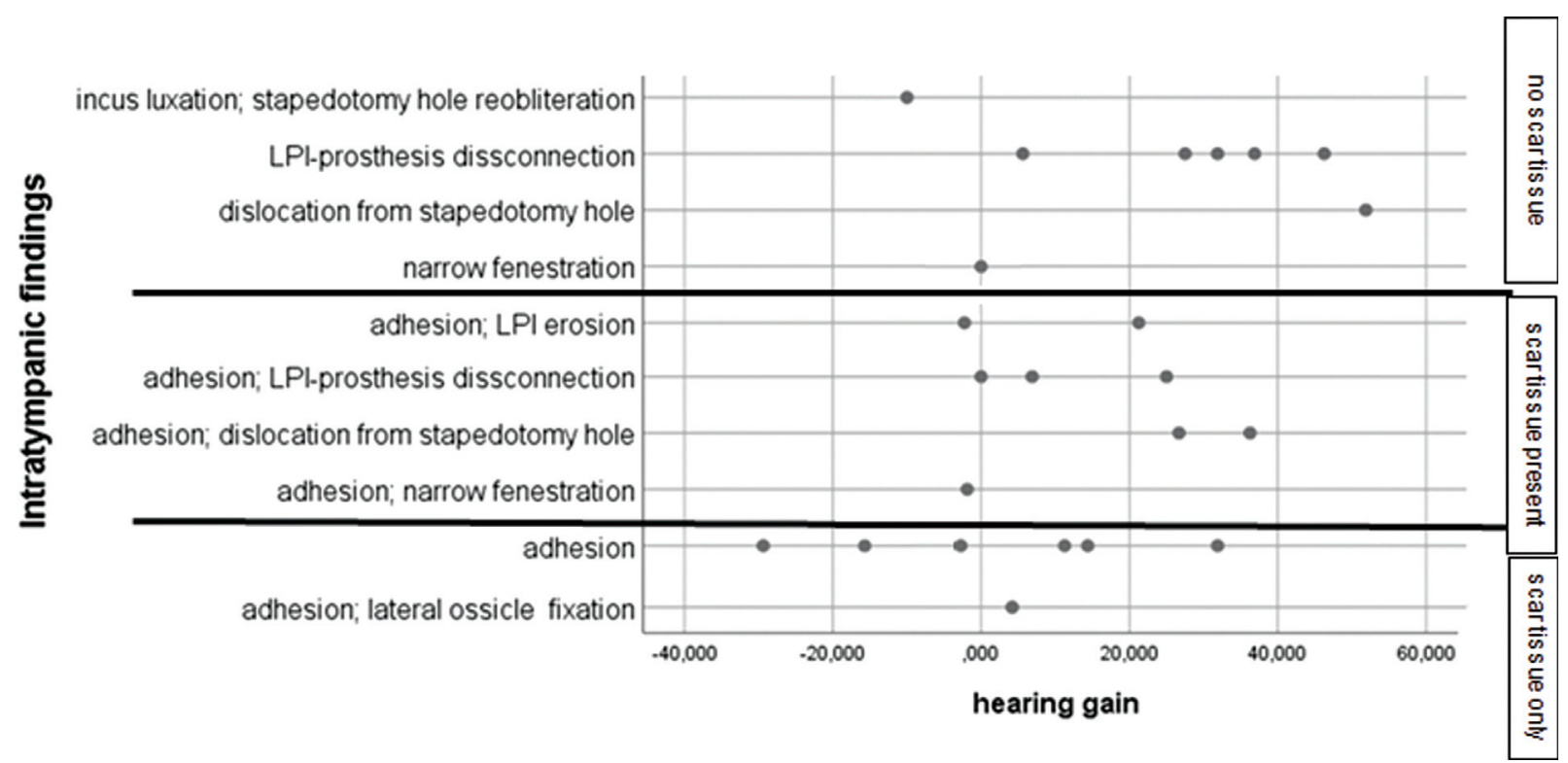

Fig. 2 Connection between hearing gain and the intratympanic finding in each revision case. The cases were classified in three groups according to intratympanic scar tissue presence. 


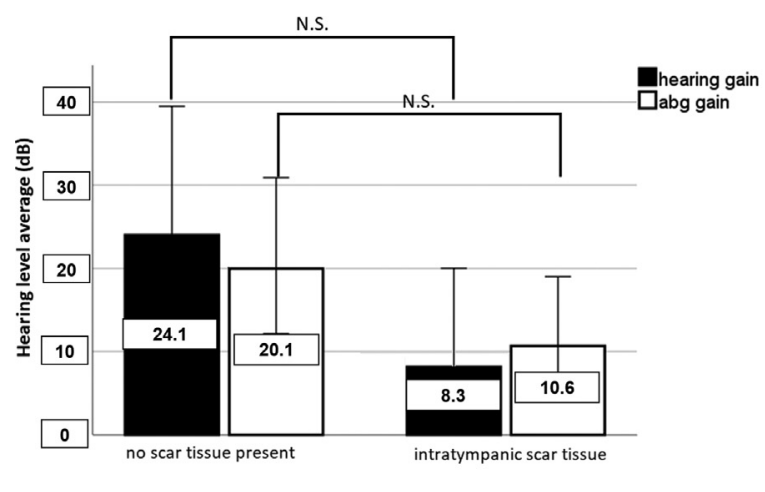

a

Emror Bars: $95 \% \mathrm{Cl}$

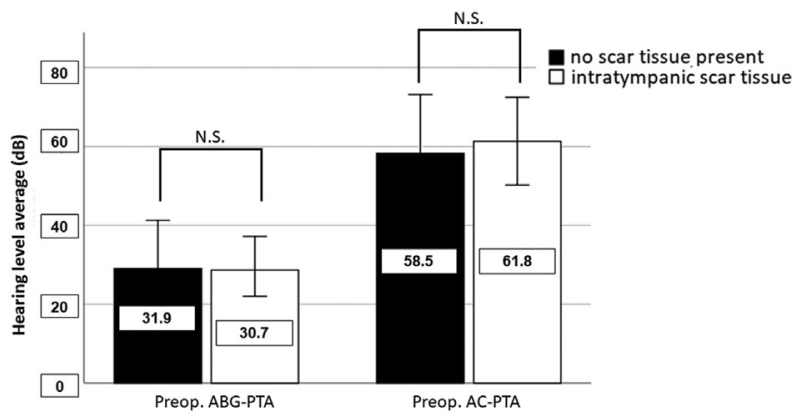

b

Error Bars: $95 \% \mathrm{Cl}$

Fig. 3 (a) ABG and hearing gain differences after revision surgery in case of scar tissue present vs. scar tissue not present. (b) ABG and AC pure tone average levels after primary stapes surgery, scar tissue present vs. scar tissue not present.

to - Fig. 2., the highest hearing gains were accomplished in cases in which the cause of failure was LPI-prothesis disconnection, while in the cases with adhesions as the primary cause of failure the hearing gains were the lowest.

Although in the presence of intratympanic adhesion mean, the ABG and hearing-gains were lower compared with other cases ( - Fig. 3a), no statistically significant difference could be found $(p=0,075 ; p=0.121)$. On this basis, TOST were performed to determine equivalence. Concerning hearing and $\mathrm{ABG}$ gains, equivalence was rejected, and the non-inferiority of the negative effects associated with intratympanic fibrosis was shown $(p=0.962$ upper tailed; $p=0.037$ lower tailed and $p=0.919 ; p=0.044$ respectively).

There was no statistically significant difference between preoperative (after primary stapes surgery) ABG and AC PTA levels across 'scar tissue' and 'no scar tissue' groups (-Fig. 3b) ( $p=0.826$ and $p=0.692$ respectively).

\section{Discussion}

Revision stapedotomy is a valuable treatment option in case of unsatisfactory hearing results after primary stapedotomy. However, our results-in consensus with other data-show that the audiological outcomes in these cases are still inferior to those of primary surgeries. From this standpoint, the efficacy of the first operation is fundamental in stapes surgery.

The experience during revision surgeries could explain the reasons behind the unsatisfactory results. In our series, periprosthetic adhesions were the leading cause behind mechanical failures. The scar tissue formation has the same negative effect on $A B G$ development after the primary surgery as any other surgical failure. At the same time, it was the most frequent intratympanic finding. Furthermore, after the revision surgery, in the presence of intratympanic adhesions, the audiological results were not superior when compared with those of the remaining cases. The difficulty of managing patients with adhesions was described previously in the literature. ${ }^{5}$

Our findings highlight the importance of the most atraumatic surgical technique, the preservation of intact intratympanic mucosa, and the prevention of postsurgical scar tissue formation.
The stapes surgery is an essential otologic procedure; however, much experience and deep surgical knowledge is needed to perform it on a high a level. The appropriate experience and surgical instruments are essential for good results, and the operation should be performed under the supervision of experienced surgeons. ${ }^{9}$

The laser-assisted stapes surgery is an increasingly widespread procedure ${ }^{10}$ with excellent audiological results. According to current knowledge, the use of potassium titanyl phosphate (KTP) and $\mathrm{CO}_{2}$ laser could be superior to other techniques, ${ }^{11}$ while promising results with new solutions such as diode laser could be found ${ }^{12}$ as well. In our practice, the use of $\mathrm{CO}_{2}$ laser is preferred during otologic surgery, due to its wide versatility and atraumatic properties. In our opinion, the main advantage of the $\mathrm{CO}_{2}$ over other laser techniques is the very low penetration depth in liquids ( $0.01 \mathrm{~mm}$ in perilymph), which is essential to avoid intraoperative inner ear damage. In our routine, during primary surgeries, beyond the footplate perforation, the posterior crus of the stapes is also vaporized with the $\mathrm{CO}_{2}$ laser single shot technique (OPAL L30; TrueScan scanner; UniMax 2000 micromanipulator; Lasram, Budapest, Hungary) before superstructure removal. The $\mathrm{CO}_{2}$ laser technique also has the capability of tympanosclerotic-lesion removal and piston crimping ${ }^{13}$ without mechanical manipulation. This noncontact surgical technique minimizes trauma of the middle ear structures and mucosa, which might prevent scar tissue formation.

The introduction of assisted or fully endoscopic stapes surgery could be another step towards minimally invasive otologic surgery. According to recent data, the audiological results are similar when compared with conventional microscopic surgery. ${ }^{14} \mathrm{~A}$ clear advantage over the microscopic technique is the better visualization due to better viewing angles, which leads to a less invasive transcanal approach by avoiding lateral atticotomy and endaural incision. The detailed exploration allowed by the endoscope is beneficial during the identification of the cause of failure. The endoscopic approach enables close visualization of the platinotomy hole and atticus, including the anterior malleolar ligament, the areas are frequently affected by scar tissue formation. Detailed magnification also allows the fine removal of the fibrous tissue. These features could be especially 
helpful in cases of middle ear malformations, in which the visualization is limited. ${ }^{15}$ On the other hand, the bleeding management and the one-handed manipulation, especially when handling the prosthesis, can be challenging. Surgeons with appropriate endoscopic ear surgery skills could consider endoscopic stapedotomy as an alternative, especially in revision cases. The better view of angles could be helpful in the identification of the exact reasons behind failure, even though the microscopic technique is still the gold standard surgical treatment for otosclerosis.

Using perioperative corticosteroid treatment to prevent postoperative fibrosis is already a topic of discussion. ${ }^{16}$ Our group was the first to describe that the above-mentioned adjuvant treatment contributes to a significantly better ABG closure after primary $\mathrm{CO}_{2}$ laser stapedotomy compared with the control group. ${ }^{17}$ Our assumption is that this contribution is manifested through the prevention of scar tissue formation.

\section{Limitations}

Due to the retrospective setting and the low number of cases, the statistical power of this study is limited, which could explain why there was no statistical difference, but noninferiority was demonstrated.

The data represents the results of a single institution and a single surgeon case study. The evaluation is based exclusively on subjective hearing tests, as the pure-tone audiometry is considered the gold standard for monitoring hearing outcomes after middle-ear surgery.

\section{Conclusion}

In our experience, the revision stapedotomy is an efficient treatment option in case of persistent $A B G$ after primary surgery. Periprosthetic fibrosis is the most common reason behind compromised audiological outcomes after stapedotomy, and the second most common failure is the prosthesis-LPI disconnection. We also concluded that the adhesion formation itself has at least the same negative effect on ABG development as any other surgical failure, although revision could be more challenging in such cases. According to these findings, the mitigation of postoperative inflammatory reactions in the middle ear is mandatory for optimal results.

\section{Bullet Point Summary:}

- Our goal was to identify the most common and most influential postoperative reasons causing persistent ABG. Our focus was on the mechanical dysfunctions in the middle ear, with special regard to postoperative adhesion formation.

- Periprosthetic adhesion formation was seen in $64 \%$ of the cases, and it was the primary cause behind the unsatisfactory hearing results in $32 \%$ of all cases.

- Periprosthetic fibrosis is the most common reason for compromised audiological outcomes after stapedotomy and has the same negative effect on ABG development as any other surgical failure, while the revision could be more challenging in these cases.

- The use of the most atraumatic surgical technique and preservation of intact intratympanic mucosa is a key factor during middle ear surgery.

\section{Ethical Approval}

The present research was approved by Semmelweis University, Budapest, Hungary. For this type of study, formal consent is not required.

Semmelweis University Regional and Institutional Committee of Science and Research Ethics (SE RKEB 159/2019.)

\section{Conflict of Interests}

The authors have no conflict of interests to declare.

\section{References}

1 Salmon C, Barriat S, Demanez L, Magis D, Lefebvre P. Audiometric Results after Stapedotomy Operations in Patients with Otosclerosis and Preoperative Small Air-Bone Gaps. Audiol Neurotol 2015; 20(05):330-336

2 Committee on Hearing and Equilibrium guidelines for the evaluation of results of treatment of conductive hearing loss. AmericanAcademy of Otolaryngology-Head and Neck Surgery Ffoundation, Inc. Otolaryngol Head Neck Surg 1995;113(03): 186-187

3 Vincent R, Sperling NM, Oates J, Jindal M. Surgical findings and long-term hearing results in 3,050 stapedotomies for primary otosclerosis: a prospective study with the otology-neurotology database. Otol Neurotol 2006;27(8, Suppl 2)S25-S47

4 Magliulo G, Cristofari P, Terranova G. Functional hearing results in revision stapes surgery. Am J Otol 1997;18(04):408-412

5 Pedersen CB. Revision surgery in otosclerosis-an investigation of the factors which influence the hearing result. Clin Otolaryngol Allied Sci 1996;21(05):385-388

6 Kanona H, Bhutta MF, Lavy J. Our approach to revision stapes surgery and the outcomes from 49 procedures at a UK tertiary centre. Clin Otolaryngol 2017;42(04):931-935

7 Andersen SA, Öhman MC, Sørensen MS. The stability of shortterm hearing outcome after stapedotomy: a prospective database study. Acta Otolaryngol 2015;135(09):871-879

8 Gurgel RK, Popelka GR, Oghalai JS, Blevins NH, Chang KW, Jackler RK. Is it valid to calculate the 3-kilohertz threshold by averaging 2 and 4 kilohertz? Otolaryngol Head Neck Surg 2012;147(01): 102-104

9 Souza JC, Bento RF, Pereira LV, et al. Evaluation of Functional Outcomes after Stapes Surgery in Patients with Clinical Otosclerosis in a Teaching Institution. Int Arch Otorhinolaryngol 2016;20 (01):39-42

10 Fang L, Lin H, Zhang TY, Tan J. Laser versus non-laser stapedotomy in otosclerosis: a systematic review and meta-analysis. Auris Nasus Larynx 2014;41(04):337-342

11 Kamalski DM, Verdaasdonk RM, de Boorder T, Vincent R, Versnel $\mathrm{H}$, Grolman W. Comparing mechanical effects and sound production of KTP, thulium, and CO2 laser in stapedotomy. Otol Neurotol 2014;35(07):1156-1162

12 Hamerschmidt R, Saab SS, Carvalho B, Carmo CD. Short-Term Audiological Results of Diode Laser in Comparison with Manual Perforation in Stapes Surgery. Int Arch Otorhinolaryngol 2018;22 (02):119-124

13 Schrötzlmair F, Suchan F, Pongratz T, Krause E, Müller J, Sroka R. Laser-assisted fixation of a nitinol stapes prosthesis. Lasers Surg Med 2018;50(02):153-157 
14 Bianconi L, Gazzini L, Laura E, De Rossi S, Conti A, Marchioni D. Endoscopic stapedotomy: safety and audiological results in 150 patients. Eur Arch Otorhinolaryngol 2020;277(01):85-92

15 Fernandez IJ, Villari D, Botti C, Presutti L. Endoscopic revision stapes surgery: surgical findings and outcomes. Eur Arch Otorhinolaryngol 2019;276(03):703-710

16 Li J, Heinz C, Zurek-Imhoff B, Heiligenhaus A. Intraoperative intraocular triamcinolone injection prophylaxis for post-cataract sur- gery fibrin formation in uveitis associated with juvenile idiopathic arthritis. J Cataract Refract Surg 2006;32(09):1535-1539

17 Székely L, Gáborján A, Dános K, et al. Mid-term evaluation of perioperative i.v. corticosteroid treatment efficacy on overall and audiological outcome following $\mathrm{CO}_{2}$ laser stapedotomy: a retrospective study of 84 cases. Eur Arch Otorhinolaryngol 2020;277 (04):1031-1038 